\title{
Identification of intrarenal fibrin deposition
}

\author{
A. M. DAVISON, D. THOMSON, MARY K. MACDONALD, J. K. RAE, \\ W. S. UTTLEY, AND A. R. CLARKSON \\ From the Department of Pathology, University of Edinburgh, the Royal Hospital for Sick Children, \\ Edinburgh, and the Medical Renal Unit, Department of Medicine, Royal Infirmary, Edinburgh
}

SYNOPSIS The site and extent of intrarenal fibrin deposition has been examined by routine histological staining, immunofluorescence, and electron microscopy in 109 patients with a wide variety of renal diseases. The findings have been correlated with the amount of urinary fibrin/fibrinogen degradation product (FDP) excretion as measured by the tanned red cell haemagglutination inhibition immunoassay. The results show that routine histological stains (Martius Scarlet Blue and Picro Mallory) are unreliable, particularly where there is mesangial accumulation of material or where the amount of fibrin deposited is small and confined to a subendothelial position. Similarly the electron microscope may overassess the degree of fibrin deposition, particularly if this is associated with the deposition of immunoglobulins and complement. There is a close relationship between the site and extent of fibrin, as detected by immunofluorescence, and the maximal urinary FDP excretion.

The role of intravascular coagulation in both experimental (Vassalli, Simon, and Rouiller, 1963) and human (McCluskey, Vassalli, Gallo, and Baldwin, 1966) renal diseases has recently attracted much attention. Lendrum, Fraser, Slidders, and Henderson (1962) described histological methods for staining fibrin of varying age and since then the techniques of immunofluorescence and electron microscopy have been applied to the assessment of fibrin deposition. It is thus important to determine the accuracy, limitations, and value of the methods currently available for the detection of fibrin in renal biopsy material.

In this study of human renal biopsies a comparison has been made between the detection of fibrin/ fibrinogen by immunofluorescence and the assessment of fibrin deposition as observed with routinely used histological fibrin stains and by electron microscopy. These results have been correlated with the urinary fibrin/fibrinogen degradation product (FDP) excretion.

\section{Methods}

Percutaneous renal biopsy was performed on 109 unselected patients, adults and children, with various renal diseases of both immunological and nonimmunological origin (Table I). The extent and distribution of fibrin as determined by routine

Received for publication 19 December 1972.

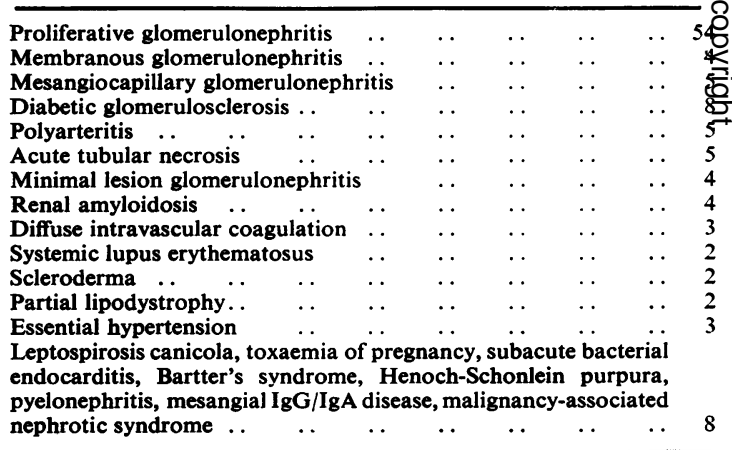

Table I Histological diagnosis of patients studied

histological stains, electron microscopy, and immunofluorescence microscopy was assessed by three independent observers. The assessments were made without knowledge of a histological or clinical diagnosis and the results compared one with the other only on completion of the study. Further, a comparison was made of the extent and distribution of intrarenal fibrin estimated by each method with the urinary excretion of FDP measured before and after the biopsy by two other independent investigators.

\section{HISTOLOGICAL STAINING}

Tissue was fixed in corrosive formol for 18 to 24 hours, sectioned at $2 \mu$, and stained by the PicroMallory V (PM) method employing acid Fuchsin 


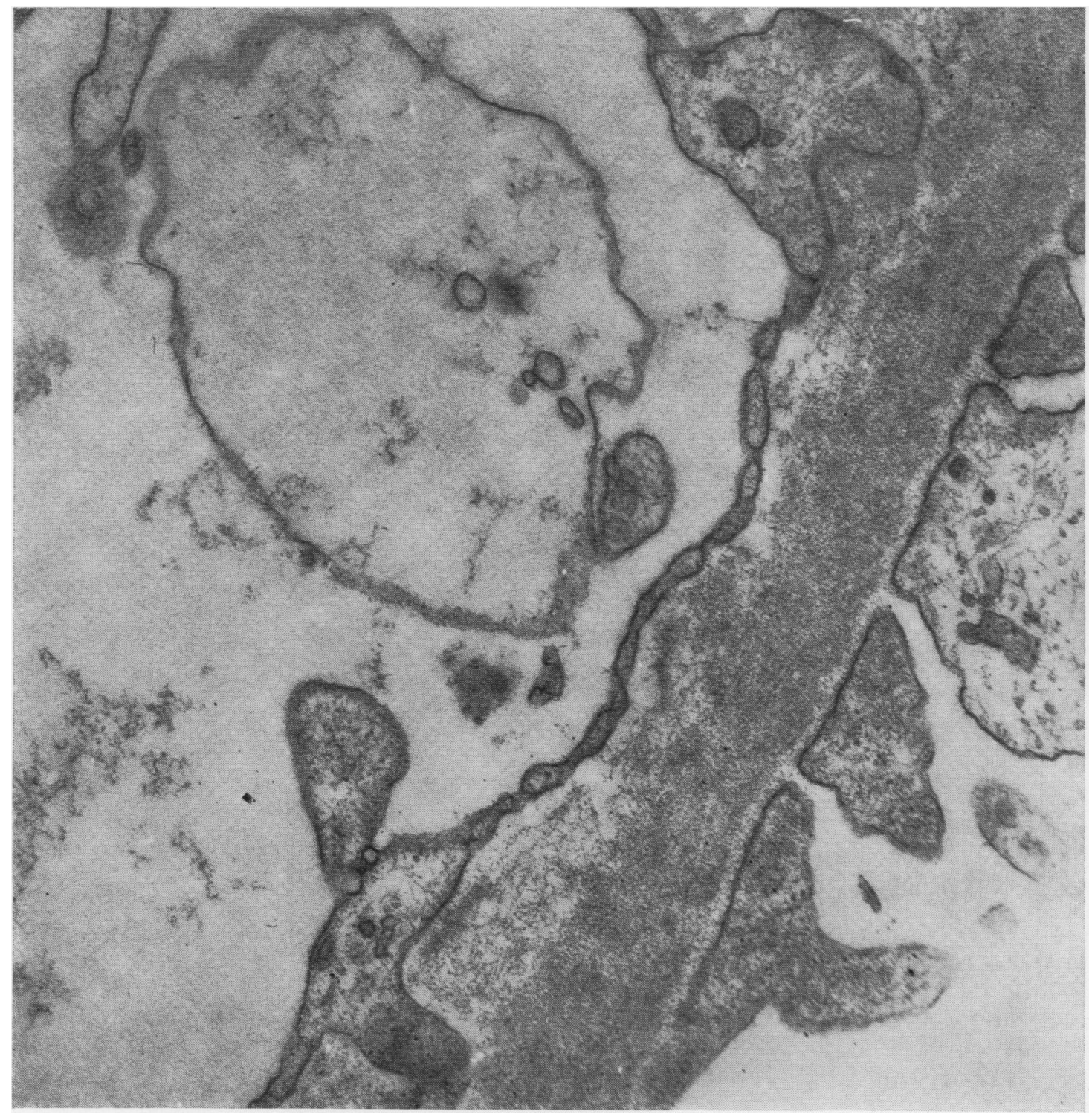

Fig. 1 Between glomerular capillary basement membrane and endothelium lies a rarefied layer in which thin fibrils are seen. Electron microscopic grade.$+ \times 30000$

(Acid Violet 19) ${ }^{1}$ and the Martius Scarlet Blue (MSB) method employing Brilliant Crystal Scarlet 6R (Acid Red 44) ${ }^{2}$ of Lendrum et al (1962).

The distribution of red staining was recorded on the following scale: 0 , no positive material; + , positive material in fewer than half of the glomeruli;

${ }^{1}$ Hopkin and Williams, Chadwell Heath, Essex.

${ }^{2}$ L. B. Holliday, Huddersfield, England.
++ , positive material in more than half of the glomeruli, and +++ , large depositis of positive material in glomeruli and also frequently in blood vessels.

Biopsies containing fewer than six glomeruli were not included in the study. Adequate material stained by MSB was obtained in 100 cases and by PM in 77 cases. 


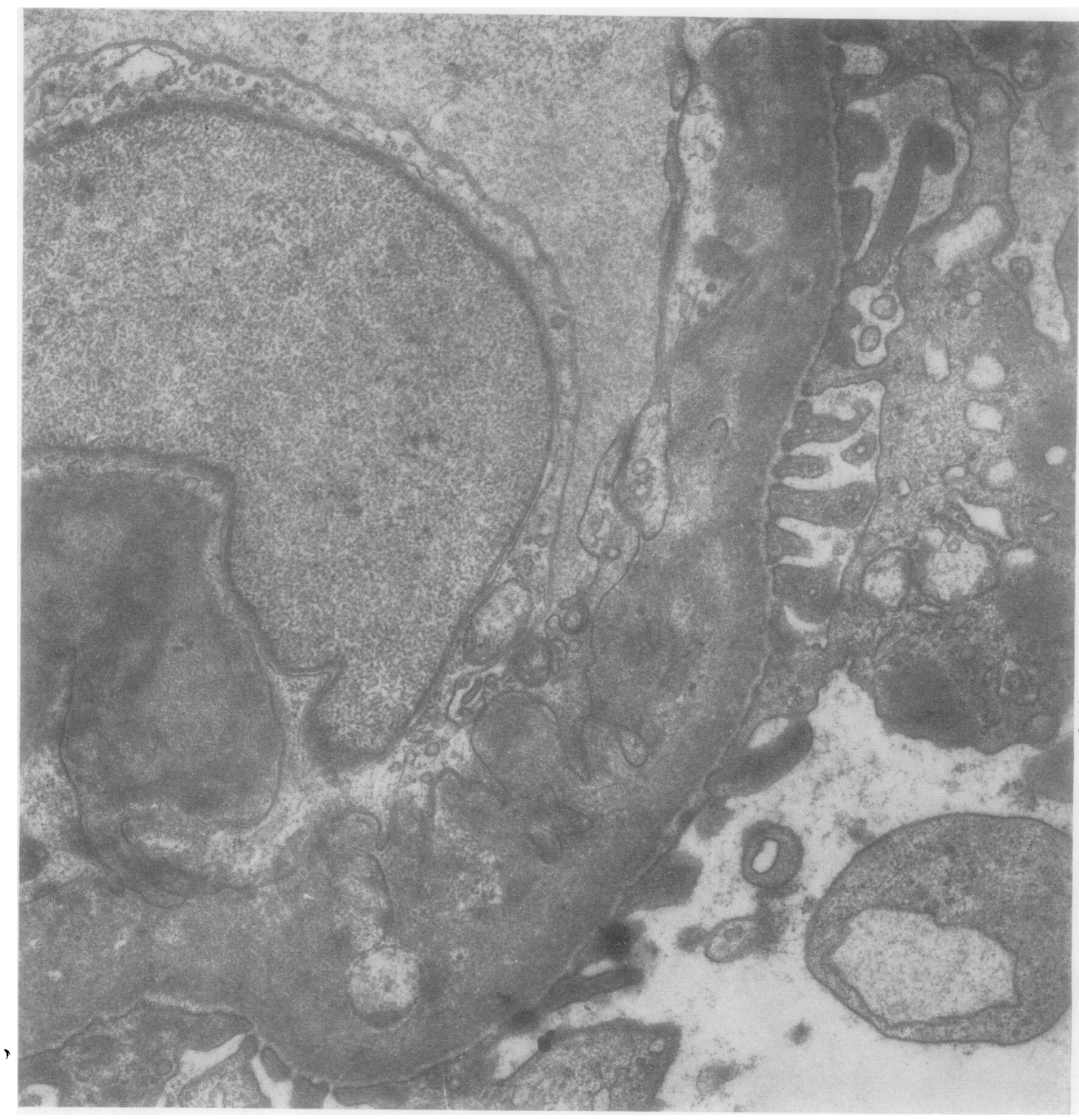

Fig. 2 Deposits of granular and fibrillar material, apparently continuous with the basement membrane, lie between membrane and endothelium, sometimes invaginating into the endothelium. Electron microscopic grade.++

ELECTRON MISCROSCOPY

Tissue was fixed in $1 \%$ osmium tetroxide, sometimes with preliminary glutaraldehyde fixation, dehydrated in graded alcohols and embedded in Araldite. Sections were cut on a Porter Blum MT2 ultratome at $50 \mathrm{~nm}$, stained with lead citrate and uranyl acetate, and viewed in an AE1 EM. 6 electron microscope.

The amount of fibrin deposited in a single glomerulus in 87 cases was assessed on the following scale:
0 , no abnormal deposits; + a rarefied layer between the basement membrane and the endothelium containing strands of osmiophilic material (Fig. 1); ++ the presence, in addition, of definite focal deposits of dark, fibrillar, or granular material between the basement membrane and the endothelium, these often showing apparent continuity with the basement membrane (Fig. 2$) ;+++$ similar but more abundant material sometimes present also 


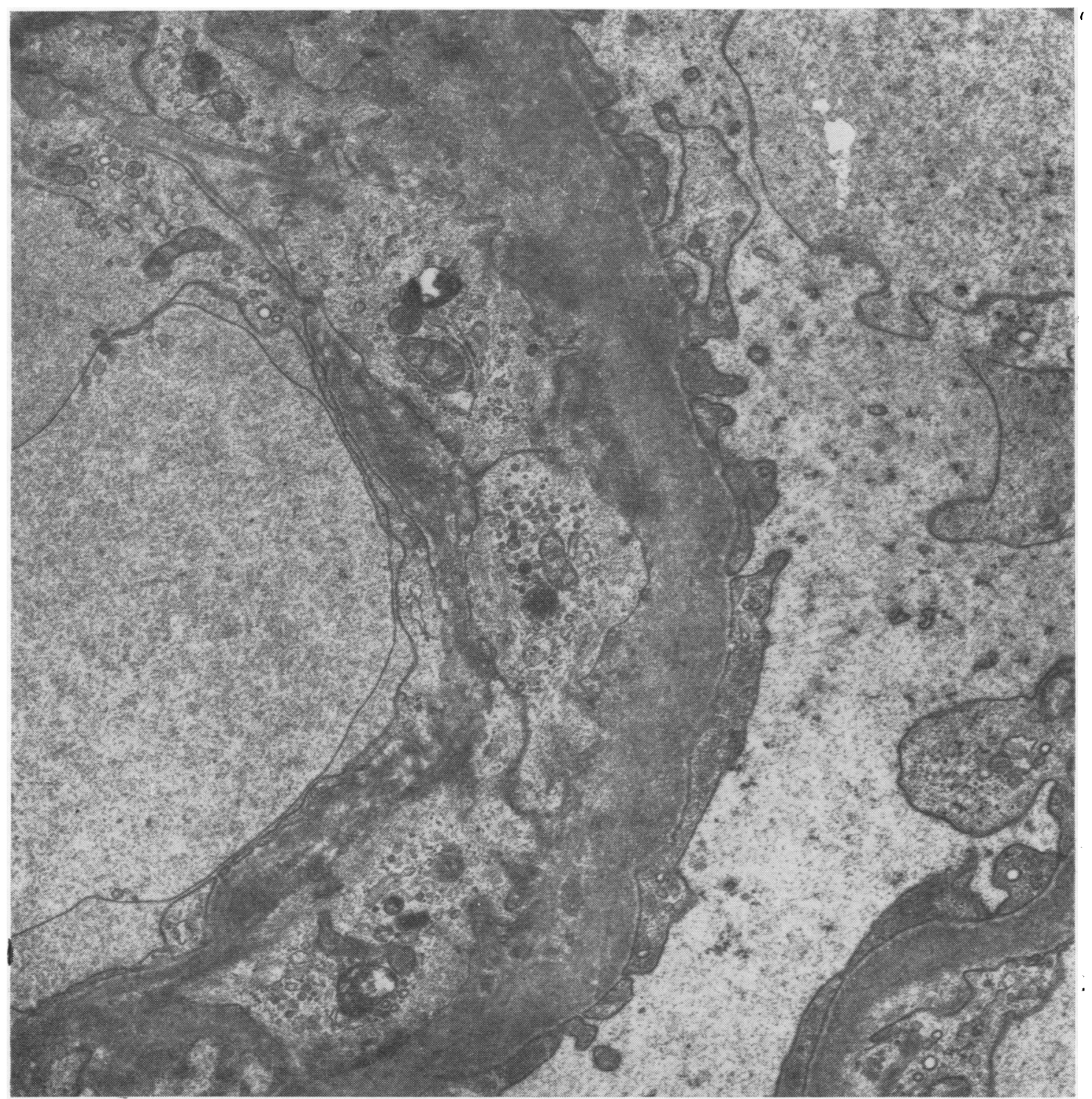

Fig. 3 The subendothelial deposits are more abundant than in Fig. 2, and are associated with reaction of the mesangial cytoplasm, which can be seen here between basement membrane and endothelium. The deposited material is in contact with the lumen through the fenestrae. Electron microscopic grade.$+++ \times 12000$

in the capillary lumen and often associated with a mesangial reaction (Fig. 3 ); ++++ complete occlusion of the glomerular capillary lumen by thrombus (Fig. 4a).

IMMUNOFLUORESCENCE MICROSCOPY Fresh tissue was 'snap-frozen' with Drikold and sectioned at 2 to $3 \mu$ in a cryostat. After fixation with
$95 \%$ alcohol for $10 \mathrm{~min}$, sections were incubated with fluorescein isothiocyanate conjugated (FITC) antihuman fibrin/fibrinogen serum (Hoechst) for 30 min in a dark, moist chamber at room temperature. Additional sections were incubated with FITC. conjugated antisera to IgG, IgM, IgA, and complement (C3).

Using this technique it is possible to decide 


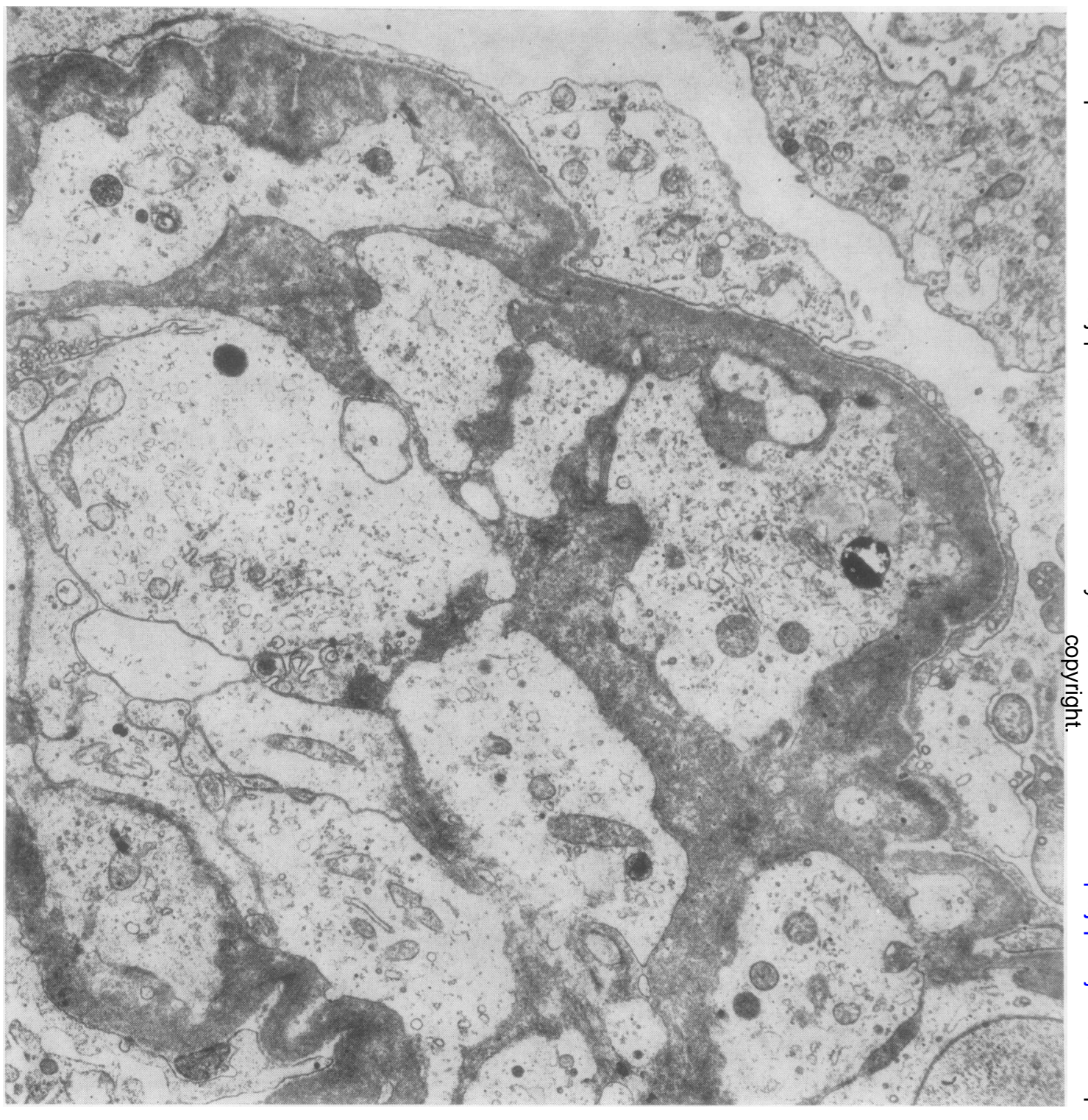

Fig. 4a Part of a glomerular capillary filled by cytoplasmic masses and intervening strands of granular and fibrillar material; this is apparently continuous with basement membrane material in places. Glomeruli in this case were found to contain large amounts of fibrin within capillaries and crescents by immunofluorescence microscopy; maximal urinary FDP excretion was greater than $20 \mathrm{mg} / \mathrm{ml}$. Electron microscopic grade.$++++ \times 6430$

whether fibrin is deposited within the glomerular capillary lumen, in the capillary wall, in mesangial regions, or in epithelial crescents. Sections containing fewer than five glomeruli were excluded from the study.

The extent and distribution of fibrin in 109 cases was recorded as follows: 0 no immunofluorescence; + positive material present in the glomerular ? capillary wall alone or mesangium alone (Fig. 5); ++ material present in the glomerular capillary wall and/mesangium (Fig. 6); +++ glomerular capillary occlusion (Fig. 7); ++++ deposits 


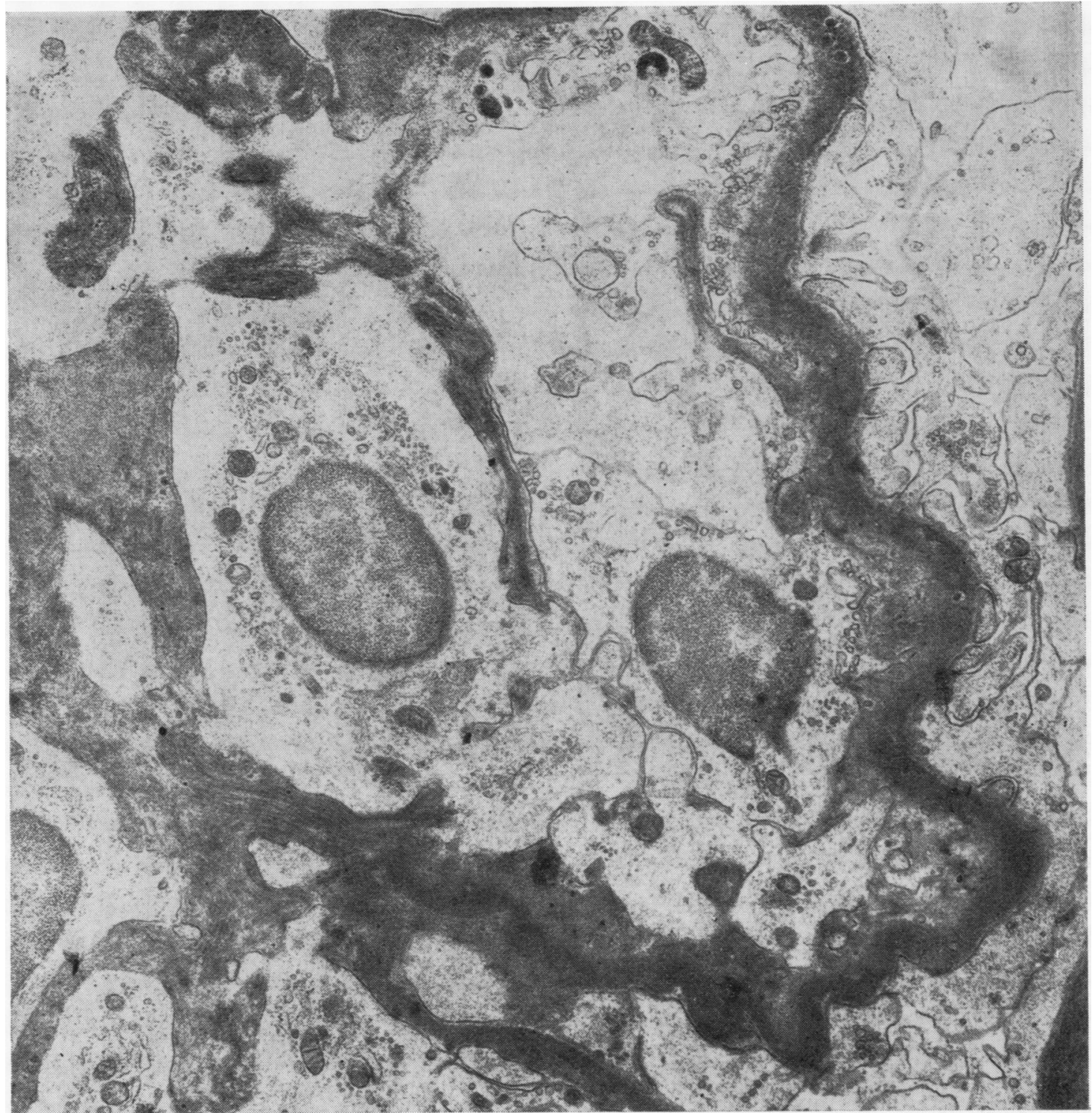

Fig. 4b The appearances are similar to Figure 4a. The glomerular capillary lumen contains granular and fibrillar material lying between cytoplasmic masses and in some foci this appears continuous with the basement membrane. By immunofluorescence microscopy the glomeruli contained a large amount of IgG, IgM, and complement $\left(C_{3}\right)$ but only small amounts of fibrin/fibrinogen; maximal urinary FDP excretion was in the range of 2 to $5 \mathrm{mg} / \mathrm{ml}$. $\times 7500$

within glomeruli and elsewhere such as epithelial crescents, interstitial spaces, or intertubular blood vessels (Fig. 8).

URINARY FIBRIN/FIBRINOGEN DEGRADATION PRODUCTS

The concentration of fibrin/fibrinogen degradation products (FDP) in the urine was measured in 94 patients by the tanned red cell haemagglutination inhibition immunoassay (Merskey, Kleiner, and Johnson 1966; Das, 1970), as modified for urine (Clarkson, Morton, and Cash, 1970) employing commercially obtained human fibrin/fibrinogen antiserum (Hoechst). Consecutive early morning 


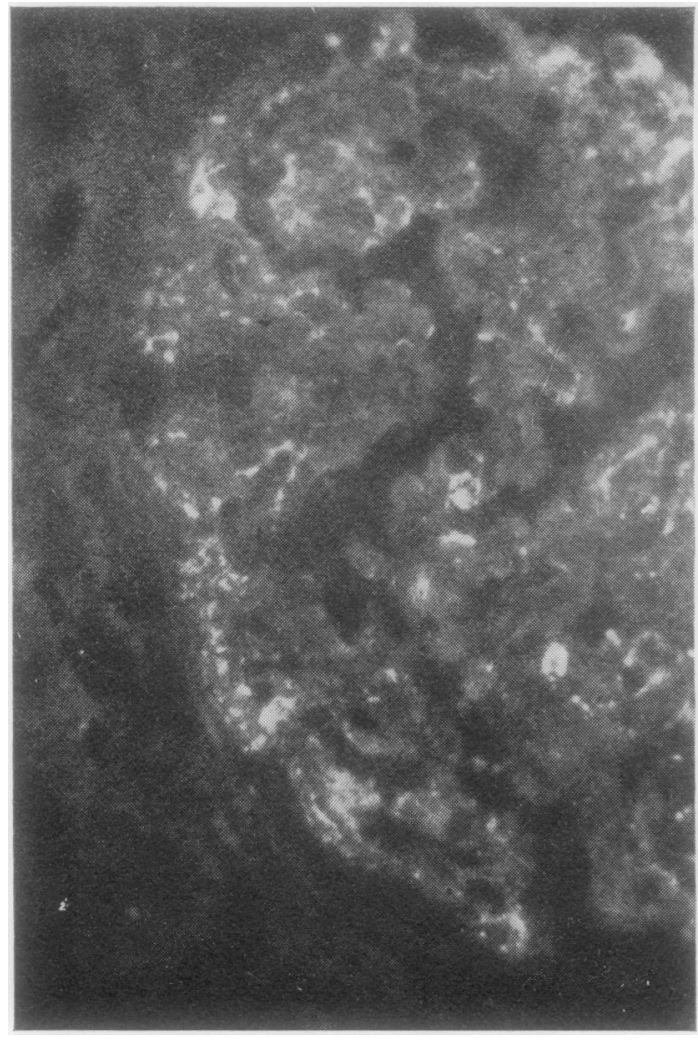

Fig. 5 Deposition of fibrin/fibrinogen within capillary walls in a patient with proliferative glomerulonephritis. Immunofluorescence grade.$+ \times 400$

urine samples collected for periods between three and 25 days before renal biopsy and between four and 150 days after biopsy were assayed and the results expressed in micrograms of fibrinogen equivalent per millilitre of urine. Maximum values obtained were compared with the results of the histological, immunofluorescence, and electron microscopic study.

Due to technical difficulties, such as inadequate biopsy material, all five possible investigations were performed in only 46 patients; 43 patients had four investigations, 15 had three, and five patients had two.

\section{Results}

\section{ROUTINE HISTOLOGICAL STAINING}

Table II shows the histological grading as estimated by 'fibrin stains' compared with the immunofluorescence microscopy grading employing specific FITC antihuman fibrin/fibrinogen serum. MSBpositive material was present in only 24 of 62 sections

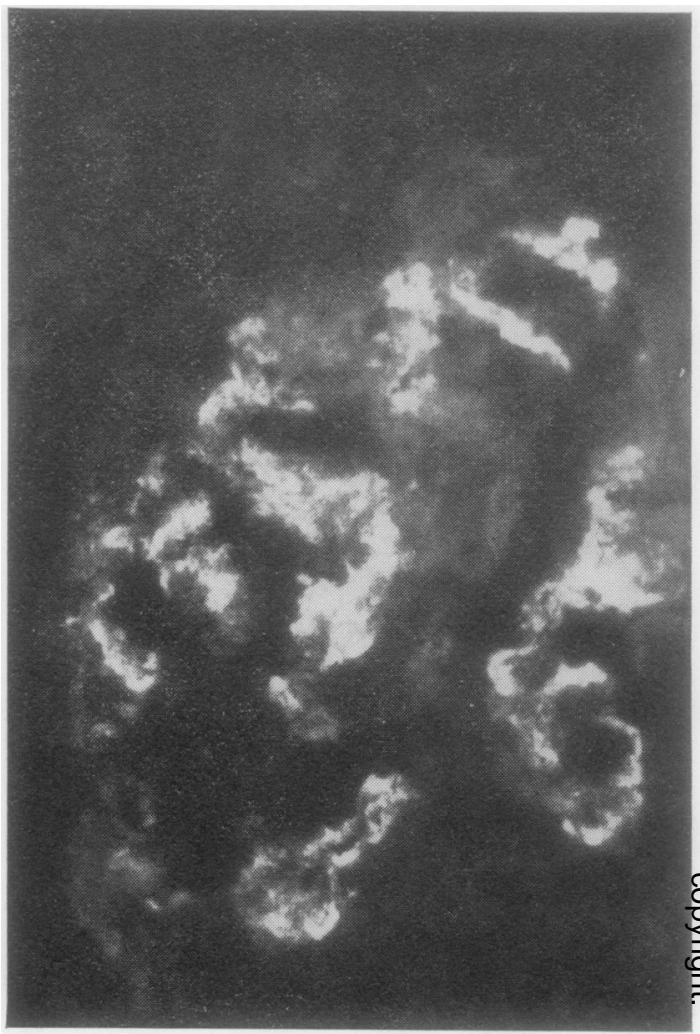

Fig. 6 Fibrin/fibrinogen within the capillary walls and mesangial regions of a patient with proliferative glomerulonephritis. Immunofluorescence grade.$++ \times 400$

in which fibrin was detected by immunofluorescence, and in nine of 36 sections in which there was no specific immunofluorescence.

Examples of positive immunofluorescence with negative MSB staining were found in all histological groups. Negative immunofluorescence and positive MSB staining occurred in glomerulonephritis, 을 amyloidosis, and diabetic glomerulosclerosis when $>$ there was predominant mesangial accumulation of

\begin{tabular}{|c|c|c|c|c|c|c|c|c|}
\hline \multirow{2}{*}{$\begin{array}{l}\text { Immuno- } \\
\text { fluorescence }\end{array}$} & \multicolumn{4}{|c|}{ Martius-Scarlet-Blue } & \multicolumn{4}{|c|}{ Picro-Mallory } \\
\hline & 0 & + & ++ & $++t$ & 0 & + & $+t$ & +++ \\
\hline 0 & 27 & 6 & 3 & & 19 & 3 & 3 & \\
\hline+ & 20 & 6 & 3 & & 15 & 3 & 4 & \\
\hline++ & 9 & 5 & 1 & & 4 & 6 & 1 & \\
\hline$+t+$ & 5 & 2 & & 1 & 2 & 3 & $i$ & 1 \\
\hline++++ & 4 & 4 & 2 & & 5 & 2 & 2 & \\
\hline
\end{tabular}

Table II Detection of fibrin by immunofluorescence microscopy and histological staining ${ }^{1}$

${ }^{1}$ See methods for details of grading 


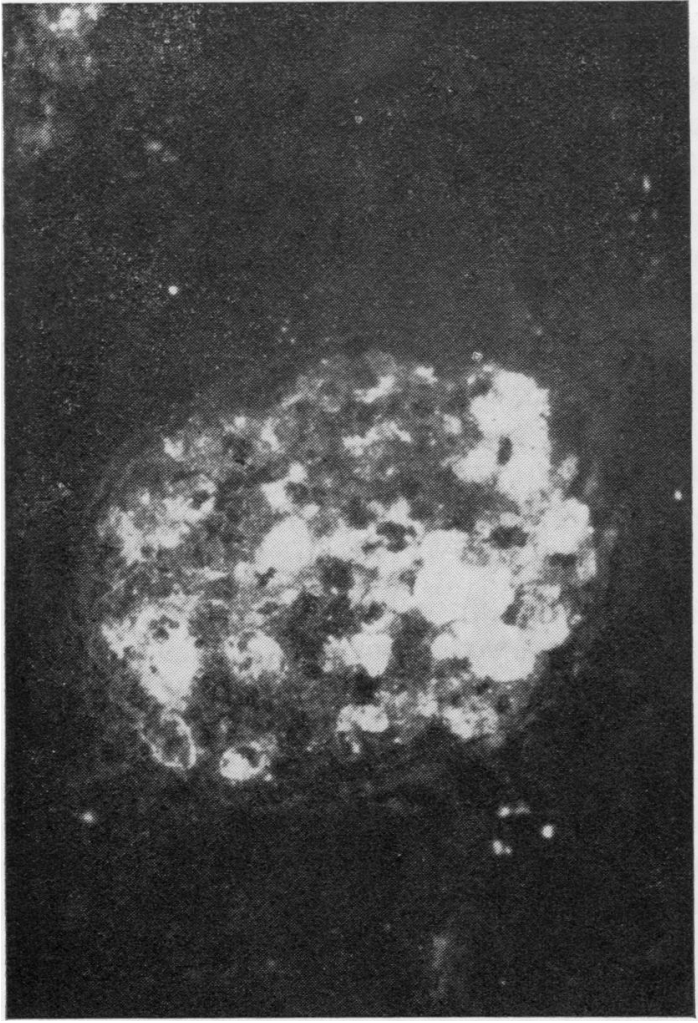

Fig. 7 Glomerular capillary occlusion with fibrin/ fibrinogen thrombi in a patient with malignancyassociated nephrotic syndrome. Immunofluorescence grade.$+++ \times 250$

material. Similar results were obtained with PM staining.

Table III shows the electron microscopic grading compared with the presence of positively staining material by routine 'fibrin stains'. The results obtained were essentially similar to those obtained with immunofluorescence except that there were fewer cases with positive histological staining in the

\begin{tabular}{|c|c|c|c|c|c|c|c|c|}
\hline \multirow[t]{2}{*}{$E M$ grading } & \multicolumn{4}{|c|}{ Martius-Scarlet-Blue } & \multicolumn{4}{|c|}{ Picro-Mallory } \\
\hline & 0 & + & ++ & $++t$ & 0 & + & ++ & +++ \\
\hline $\mathbf{0}$ & 6 & & 2 & & 4 & 1 & 1 & \\
\hline+ & 14 & 5 & & & 12 & & 3 & \\
\hline$+t$ & 17 & 2 & & & 12 & 2 & 2 & \\
\hline+++ & 13 & 7 & 2 & & 8 & 10 & 2 & \\
\hline$++t+$ & 4 & 5 & 1 & & 2 & 2 & 2 & \\
\hline
\end{tabular}

Table III Electron microscopic grading of fibrin and histological staining ${ }^{1}$

${ }^{1}$ See methods for details of grading

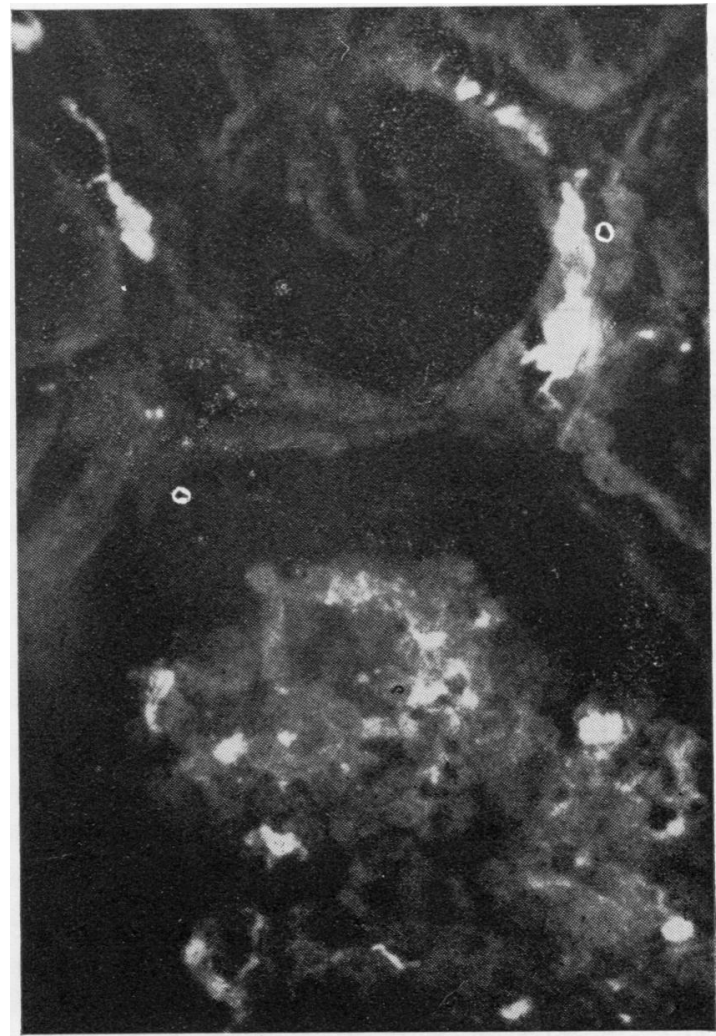

Fig. 8 Fibrin/fibrinogen within glomerular capillary walls and also peritubular capillaries in a patient with haemolytic uraemic syndrome. Immunofluorescence grade $++++. \times 400$

absence of electron microscopic detection of fibrin. Material positive with MSB was present in one case of acute renal failure and one of amyloidosis in which no fibrin was detected by electron microscopy; PMpositive material was present in one case of resolving proliferative glomerulonephritis and one of amyloidosis in which no fibrin was detected by electron microscopy.

There were 73 cases with both adequate MSB and PM staining. In 53 cases there was concordance between these two methods, while in 12 there was positive staining by PM only and eight were positive by MSB only.

\section{ELECTRON MICROSCOPY}

Table III shows the electron microscopic grading compared with MSB and PM staining.

Table IV shows the electron microscopic assessment compared with the maximum urinary FDP excretion. The correlation between these two 


\begin{tabular}{|c|c|c|c|c|c|c|}
\hline \multirow{2}{*}{$\begin{array}{l}\text { Glomerular } \\
\text { Fibrin } \\
\text { Electron } \\
\text { Microscopic } \\
\text { Grading }\end{array}$} & \multicolumn{6}{|c|}{ Maximum Urine FDP Concentration $(\mu \mathrm{g} / \mathrm{ml})$} \\
\hline & $0-1$ & $1-2$ & $2-5$ & $5-10$ & $10-20$ & $>20$ \\
\hline $\mathbf{0}$ & 6 & 2 & & 1 & & \\
\hline+ & 5 & 4 & 6 & 1 & & 1 \\
\hline++ & 5 & 3 & 2 & 2 & 1 & 2 \\
\hline$++t$ & 3 & 2 & 1 & 4 & 3 & 4 \\
\hline+++ & 1 & 1 & 6 & & 1 & 2 \\
\hline
\end{tabular}

Table IV Relation between urinary fibrin/fibrinogen degradation products and extent of intraglomerular fibrin deposition as judged by electron microscopy (69 patients) ${ }^{1}$

${ }^{1}$ See methods for details of grading

measurements is good ( $P<0.001)$ but with a slight tendency towards overassessment by electron microscopy, eg, eight patients were considered as ++++ by electron microscopy but had a urinary FDP concentration of $5 \mu \mathrm{g} / \mathrm{ml}$ or less. In these patients fibrin was present, but in no case was it assessed as greater than ++ by immunofluorescence. In addition these patients had considerable deposits of immunoglobulin, particularly IgG and IgM, and complement $\left(\mathrm{C}_{3}\right)$ (Fig. $4 \mathrm{~b}$ ). The histological diagnosis of these patients was proliferative glomerulonephritis in six and mesangiocapillary glomerulonephritis in one; one patient had clinical partial lipodystrophy. Of the two patients with high urinary FDP concentration but little electron microscopic evidence of fibrin deposition, one had acute tubular necrosis (urine FDP $5-10 \mu \mathrm{g} / \mathrm{ml}$; EM grading 0) and the other had a mild proliferative glomerulonephritis (urine FDP > $20 \mu \mathrm{g} / \mathrm{ml}$; EM grading +).

Table $\mathrm{V}$ shows the relationship between the electron microscopy and the immunofluorescence findings. There is a wide distribution, again with evidence of overassessment by electron microscopy. Twelve patients graded as ++ or greater by EM had no fibrin detectable by immunofluorescence; the histological diagnosis of this group was diabetes mellitus (5), proliferative glomerulonephritis (3), membranous glomerulonephritis (2), polyarteritis (1),

\begin{tabular}{llllll}
\hline $\begin{array}{l}\text { Immuno- } \\
\text { fluorescence }\end{array}$ & \multicolumn{4}{l}{ Electron Microscopic Grading } \\
\cline { 2 - 6 } & 0 & + & ++ & +++ & ++++ \\
\hline 0 & 8 & 7 & 6 & 5 & 1 \\
+ & 1 & 8 & 8 & 5 & 4 \\
++ & 2 & 2 & 3 & 4 & 5 \\
+++ & 1 & 1 & 5 & 1 \\
++++ & & 1 & 2 & 5 & 1 \\
\hline
\end{tabular}

Table V Detection of fibrin by immunofluorescence microscopy and electron microscopy (86 patients)

${ }^{1}$ See methods for details of grading and scleroderma (1). Only four of this group, two membranous, one polyarteritis, and one focal $\overrightarrow{0}$ proliferative, showed any evidence of immuno- globulin deposition, but in all four the presence of $\overrightarrow{\vec{\rho}}$ IgM could be demonstrated. Nine patients were graded ++++ by electron microscopy but showed only + or ++ fibrin deposition by immunofluorescence; seven of these had proliferative glomerulonephritis, one mesangiocapillary glomerulonephritis, and one partial lipodystrophy. However, $\infty$ in all the presence of IgG could be shown, IgM in $\vec{O}$ eight and complement $\left(\mathrm{C}_{3}\right)$ in seven.

IMMUNOFLUORESCENCE MICROSCOPY

Table VI shows the immunofluorescence grading of intrarenal fibrin deposition and the maximum o urinary FDP concentration. The relationship be-? tween these two methods is highly significant $\vec{N}$ (P $<0.001)$. Two patients with an immunofluore- 0 scence rating of ++ and with a urinary FDP con- $\supset$ centration of 0 to $1 \mu \mathrm{g} / \mathrm{ml}$ were receiving therapy, $T$ one with corticosteroids and one with indomethacin. $\frac{\mathbb{D}}{\sigma}$ One patient with a urinary FDP concentration of 5 to $10 \mu \mathrm{g} / \mathrm{ml}$ and no immunofluorescence for fibrin had acute renal failure due to infection with Lepto- $\overrightarrow{0}$ spirosis canicola.

\begin{tabular}{|c|c|c|c|c|c|c|}
\hline \multirow{2}{*}{$\begin{array}{l}\text { Fibrin/ } \\
\text { Fibrinogen } \\
\text { Immuno- } \\
\text { fluorescence }\end{array}$} & \multicolumn{6}{|c|}{ Maximum Urine FDP Concentration $(\mu g / m l)^{1}$} \\
\hline & $0-1$ & $1-2$ & $2-5$ & $5-10$ & $10-20$ & $>20$ \\
\hline $\begin{array}{l}0 \\
+ \\
++ \\
+++ \\
++++\end{array}$ & $\begin{array}{r}20 \\
9 \\
2\end{array}$ & $\begin{array}{l}8 \\
6\end{array}$ & $\begin{array}{l}1 \\
7 \\
9 \\
1\end{array}$ & $\begin{array}{l}1 \\
1 \\
3 \\
3 \\
2\end{array}$ & $\begin{array}{l}2 \\
3 \\
3\end{array}$ & $\begin{array}{l}2 \\
6\end{array}$ \\
\hline
\end{tabular}

Table VI Relation between urinary fibrin/fibrinogen degradation products and immunofluorescence to fibrin/ fibrinogen ( 88 patients)

${ }^{1}$ See methods for details of grading

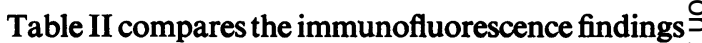
with routine histological stains. Table $\mathrm{V}$ shows the $\frac{\mathrm{T}}{\mathrm{O}}$ immunofluorescence findings compared with the electron microscopic grading.

URINARY FDP

Table VI compares the maximal urinary FDPW concentration with the immunofluorescence findings. The most consistently elevated values were found읃 where fibrin was seen in extraglomerular positions, $\mathbb{\Phi}$ ie, within crescents, interstitium, blood vessels, or ${ }^{-}$ tubules. In all patients with glomerular capillary deposition of fibrin the abnormal FDP excretion was $\frac{\text { Pे }}{\mathrm{C}}$ cyclical, with varying intervals of minimal excretion $\frac{?}{9}$ 
between periods of more marked abnormality (two to 25 days). This was most apparent in patients with proliferative glomerulonephritis.

Table IV shows the maximal urinary FDP concentration compared with the electron microscopy findings.

\section{Discussion}

The intravascular deposition of fibrin is a dynamic process beginning with the alteration of fibrinogen by thrombin, progressing to the formation of fibrin, and eventually to the production of various degradation products. It would seem unlikely, therefore, that the presently available routine histological stains would have an equal affinity for the wide variety of forms through which fibrin passes. In this study we have found a poor relationship between histological stains and the immunofluorescent detection of fibrin, though this may be due to some extent to the fact that we have not carried out the prolonged fixation for two to three weeks in aqueous mercuric chloride or the degreasing recommended by Lendrum and his coworkers (1962). We omitted these steps because (1) the quantity of material we employed, obtained by needle biopsy, was small and therefore fixed readily, and (2) in most instances the clinical necessity for diagnosis merited more rapid processing. False positive histological results occur in diseases where there is predominantly mesangial accumulation of material while the lack of immunological characteristics of fibrin could be due to the destruction of antigenic sites on the fibrin molecule following phagocytosis by the mesangial cell; it is probable that the accumulated material actually contains little or no fibrin. False negative results may occur because of different sensitivities of the methods employed; it is unlikely that histological stains can detect small amounts of fibrin deposited in a subendothelial position as seen in grade + by electron microscopy or grade + by immunofluorescence microscopy. As in most renal diseases continuation of the coagulation process occurs until remission, spontaneous or drug induced, all stages of fibrin formation and removal are likely to be found. Thus the use of dyes which predominantly stain early or late fibrin is likely to be of little value, although it did seem that the early staining PM possessed a closer relationship to immunofluorescence microscopy than MSB.

For years it was believed that the detection of fibrin by electron microscopy was dependent upon observing fibrillar material with a characteristic periodicity of approximately $200 \AA$. However, it has been shown that fibrin may not have this characteristic appearance. In addition, although fibrin and the largest of the degradation products may exist as filamentous fibres with characteristic periodicity, the other intermediate and end products appear as rudimentary fibrils or amorphous granular structures. This leads to difficulty in the interpretation of electron micrographs; in the present study there is a reasonable relationship between the identification of fibrin by the electron microscope and the excretion of FDP in the urine, but there is a tendency to overdiagnosefibrin by electron microscopy compared with identification by immunofluorescence microscopy, and this is probably explained by the similar appearance of certain products of fibrin formation and degradation and of other protein deposits. This was confirmed by finding considerable deposits of immunoglobulins and complement in those biopsies scored as ++ or greater by electron microscopy and containing FDP in the urine of less than $5 \mu \mathrm{g} / \mathrm{ml}$ (Figs. $4 \mathrm{a}$ and $\mathrm{b}$ ). In addition, of the eight patients with diabetes mellitus five were graded as ++ or greater by electron microscopy, but showed no specific immunofluorescence. With the currently available techniques, the electron microscope cannot clearly distinguish the exact nature of protein deposits; certain features such as the apparent continuity of deposits with the basement membrane and the presence of a rarefied and slightly fibrillar subendothelial layer suggest that the material present is fibrin, but definite identification is not possible without typical periodicity.

The immunofluorescence microscopy grading of fibrin deposits is not strictly comparable with the electron microscopic assessment. However, it probably represents a more accurate estimation of the distribution of intrarenal fibrin, since it is possible to visualize several glomeruli and other important structures such as blood vessels. Immunofluorescence grading depends more on distribution than on a quantitative assessment. This is necessarily so as methods of immunofluorescence microscopy quantitation on tissue sections are difficult, time consuming, and unlikely to reveal much additional information when employed on a routine basis.

There is a very close agreement between the fibrin distribution as assessed by immunofluorescence and the maximal excretion of FDP in the urine. Where the fibrin deposits were confined to the glomerulus and present only in the capillary wall or mesangium, there was a slight increase in urine FDP excretion. This was presumably due to a limited activation of the coagulation system with adequate removal by lysis and phagocytosis. However, where fibrin was detected occluding the capillary lumen, in epithelial crescents, or in peritubular capillaries, urinary FDP concentrations were considerably increased. Most patients in this group were found to have progressive 
proliferative glomerulonephritis or acute ischaemic renal failure. Since the urinary excretion of FDP is episodic in many cases of proliferative glomerulonephritis, it is important to measure daily urine concentrations for up to 25 days to ensure that positive cases are not missed. The serial measurement of urinary FDP provides a good method of following the state of intrarenal fibrin deposition and therefore the natural history or response to therapy of the basic pathological process.

This study therefore confirms the involvement of the coagulation system in a wide variety of renal diseases. It has demonstrated the limitations of the routine histological stains and of electron microscopy in assessing the degree of fibrin deposition in renal biopsy specimens. The close relationship between the intrarenal fibrin as detected by specific immunofluorescence and the maximum urinary FDP excretion confirms the value of the latter technique in following patients over considerable time intervals. In addition it suggests that the distribution of fibrin, as viewed by immunofluorescence, may be of more significance than an estimate of the total amount deposited.

We are grateful for the technical assistance of Miss Leslie Taylor, Mr Robert Donaldson, Mr James Barclay, and Mrs Heather Maxwell.
Financial support has been given by the Lawson Tait Medical and Scientific Trust, the Scottish Home and Health Department and the Scottish Hospital Endowments Research Trust, the Winston Churchill Memorial Trust of Australia, and the Research Endowment Fund of the Royal Hospital for Sick Children, Edinburgh.

We wish to thank the many physicians under whose care these patients were investigated. We are grateful to Drs J. S. Robson and J. D. Cash for advice and encouragement with this study.

\section{References}

Clarkson, A. R., Morton, J. B., and Cash, J. D. (1970). Urinary fibrin fibrinogen degradation products after renal homotransplantation. Lancet, 2, 1220-1223.

Das, P. C. (1970). Assay of seru: agglutination-inhibition of coated erythrocytes. J. clin. Path., 23, 299-303

Lendrum, A. C., Fraser, D. S., Slidders, W., and Henderson, R. (1962). Studies on the character and staining of fibrin. J. clin. Path., 14, 401-413.

McCluskey, R. T., Vassalli, P., Gallo, G., and Baldwin, D. S. (1966) An immunofluorescent study of pathogenic mechanisms in glomerular diseases. New Engl. J. Med., 274, 695-701.

Merskey, C., Kleiner, G. J., and Johnson, A. J. (1966). Quantitative estimation of split products of fibrinogen in human serum, relation to diagnosis and treatment. Blood, 28, 1-18.

Vassalli, P., Simon, G., and Rouiller, C. (1963). Electron microscopiô study of glomerular lesions resulting from intravascular fibri formation. Amer. J. Path., 43, 579-617. 